\title{
Design and Implantation of a Voluntary Reading Mobile Application for People who are Visually Impaired
}

\author{
${ }^{1}$ Afnan AbouElwafa*, ${ }^{1}$ Nadia Selim,,${ }^{1}$ Hanan Alateeq, ${ }^{1}$ Dalal Alzaid, ${ }^{2}$ Shiroq Al-Megren, and ${ }^{1}$ Heba Kurdi \\ ${ }^{1}$ Computer Science Department, King Saud University, Riyadh, Saudi Arabia \\ ${ }^{2}$ Information Technology Department, King Saud University, Riyadh, Saudi Arabia
}

\begin{abstract}
This paper proposed an application that supports visually impaired users in the community by providing voluntary audio support for readable content. The application, Basirah, allows visually impaired users to post audio request for reading text. Volunteers are able to view these requests and offer their response as recorded audio links. The application is developed for iOS devices and supports requests in English and allows for multi-lingual responses for volunteers. This paper presents Basirah and walks through the analysis, design, and testing phases of development. Preliminary testing was carried out on Basirah, where it has shown promising results and contribution.
\end{abstract}

Key words: Visually impaired, reading, mobile application, accessibility.

\section{Introduction}

The World Health Organization reports that 285 million people around the world are visually impaired [1]. This segment of society relies on other senses to support their literacy development and reading, principally hearing. While several efforts attempt to cater to their audio needs via textto-speech applications and audiobooks, there still remains a gap that does not take into account the needs of visually impaired users. The majority of applications available to convert text into digital audio that does not reflect the unique properties of the human voice, such as appropriate pauses and inflictions. Moreover, current applications may only offer support for certain text types and thus limit the range of reading materials available to users who are visually impaired.

This paper presents Basirah, an iOS application for Apple devices that bridges sighted and visually impaired readers in the community to support more natural audio descriptions and text types. The paper describes the first phases of the application development, which includes the analysis, design, and implementation phases. The result of this process is a fully-functioning application that has shown promising results in preliminary testing.

This paper describes the implementation process of Basirah. In Section 3 requirements are gathered and analyzed to extract features the application's features. Section 4 presents the design of Basirah. The system implementation is described in Section 5. The final section, Section 6, describes the testing process starting with unit component and ending with an overall system evaluation. The

*Corresponding author: Afnan AbouElwafa, Address: Computer Science Department, King Saud University, 12371 Riyadh, Saudi Arabia. E-mail address: 436203229@student.ksu.edu.sa, Phone: +966-11-805-7867 
paper finally concludes and presents some future planned work.

\section{Related Work}

The proposed solution falls within the context of electronic reading and applications accessible to people who are visually impaired. In the proposed application, visually impaired users are able to request what they wish to listen to rather than being limited to what is provided in the application's library or type of text. The developed application should also be available as an open-source contribution that is available for free download and usage. The utilization of a human voice is an important feature that conveys natural voice characteristics, such as pausing in the correct places, changes in tone, and emotional impact.

Several applications share some of these properties to support accessible reading to people who are visually impaired or dyslexic. KNFB Reader is an application that aims to support people who are visually impaired or dyslexic by allowing them to take a photo from any text surface and reads it out loud (see Fig. 1). The KNFB Reader application is available for iOS, Android, and Window 10 devices. However, application is relatively costly and the text is voiced using an automated voice.

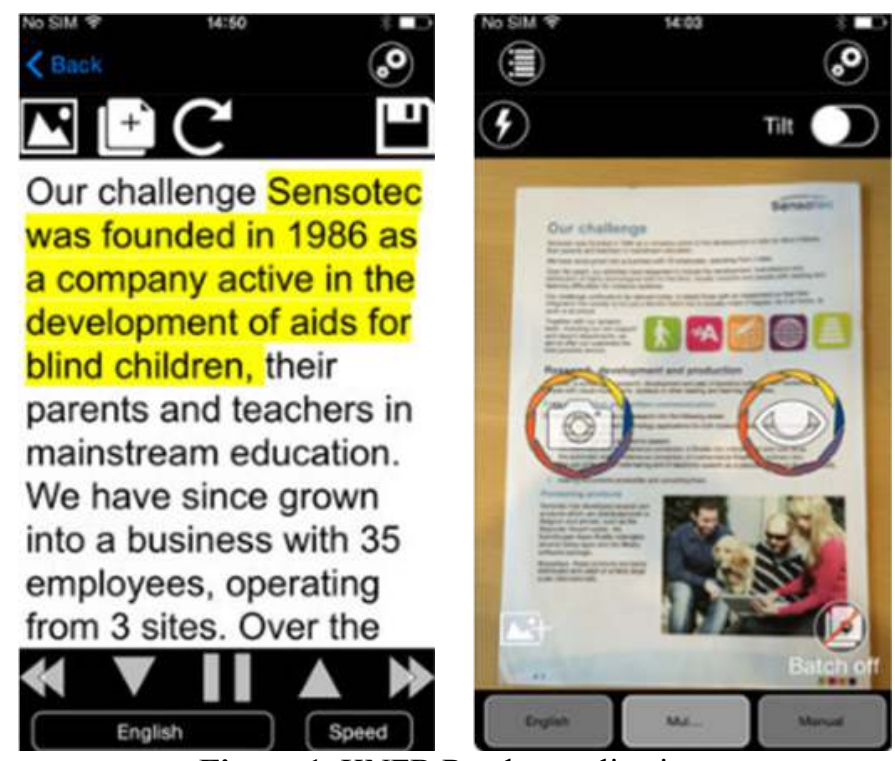

Figure 1. KNFB Reader application.

Read2Go is another application that share similarities with the developed solution. The application is an electronic book reader that is only available on iOS devices (see Fig. 2). Read2Go is primarily developed for users who are blind, have low visions, or suffer from learning or physical disabilities [2]. In the application, the user can search, download, and listen to books available on the bookshare library and daisy books. As a result, not all types of text are supported. To use the application, users must have a booshare membership that is only available for free for United States studies and organisations. The application is cheaper than that of KNFB reader but is not free. 


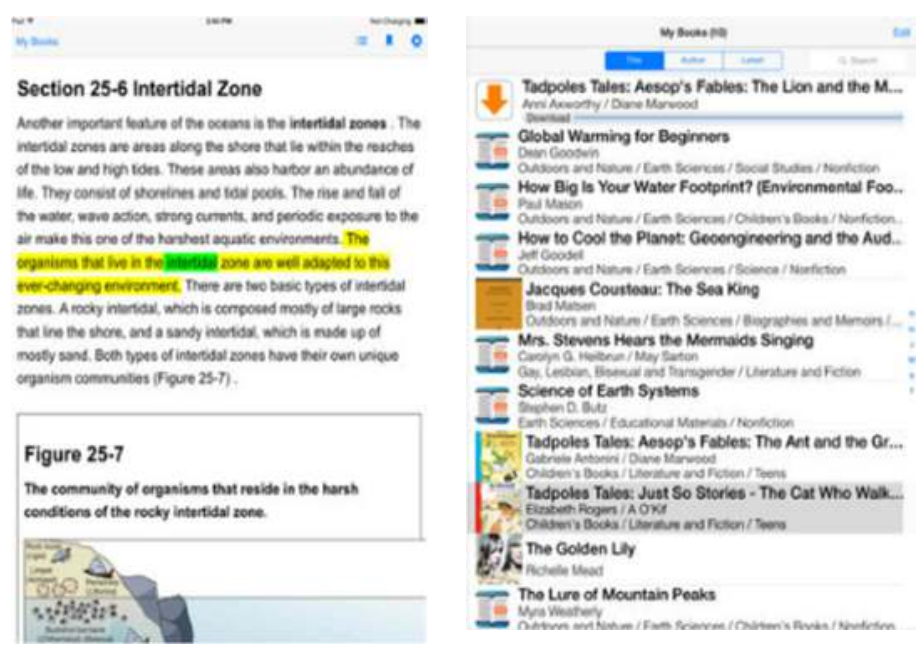

Figure 2. Read2Go application.

AlManarah Library is an Arabic based application that is developed by the Al-Manara Charitable Society [3]. The digital library aims to universally provide a digital library of books, articles, and stories (see Fig. 3). The applications also offers support for users who are visually impaired. Furthermore, users who are visually impaired are given a free premium subscription. This is not the case for sighted users that are expected to pay.
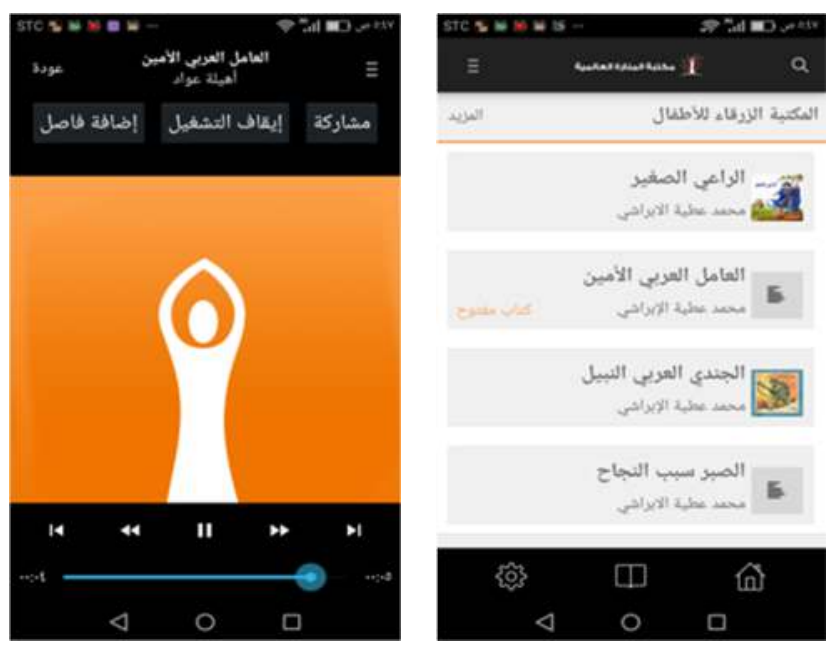

Figure 3. AlManarah library application.

Several other applications that are considered to be electronic books can also be found. This group of applications do not offer support for users who are visually impaired or dyslexic. Such applications include, Alrawi, EqraaLee, and Arabic audio books. These applications often share similar functionalities, where users can search and listen for books. 
Table 1. Comparison between related work applications

\begin{tabular}{lcccc}
\hline Support/Application & Ability to request & Any type of text & Free license & Human voice \\
\hline KNFB Reader & Yes & Yes & No & No \\
Read2Go & No & No & No & No \\
AlManarah & No & No & Yes & Yes \\
Alrawi & No & No & No & Yes \\
EqraaLee & No & No & No & Yes \\
Arabic audio books & No & No & Yes & Yes \\
Basirah & Yes & Yes & Yes & Yes \\
\hline
\end{tabular}

Table 1 summaries the application reported here to compare their relevant features. The developed application, Basirah, has four main advantages that rarely appear on other applications. First, they give user who are visually impaired the ability to request whatever text they need to listen to and not only to what is provided by the application. Second, Basirah does not limited users to a certain text type. That means it is not limited to books, articles, or stories. Third, the developed application will be available for free. Finally, Basirah aims to build linked between volunteers and people who are visually impaired to help increase the community's awareness of the needs of the visually impaired.

\section{System Analysis}

The waterfall model was utilized to developed the applications, Basirah, as the model is maintainable and easy to use. Furthermore, the application has a small scope with very low risks and clear requirements. These characteristics support the utilization of the waterfall model for development.

\subsection{Requirement Analysis}

Two approaches were used to collect the software requirements: informal interviews and comparison with related software. Several people who are visually impaired were asked a series of questions concerning their problems with reading applications. The interviewees were prompted to share their needs and likes to ensure an application that offer supports for users who are visually impaired. The other method was used to survey the application area of reading applications based on the interview outcomes.

\subsection{Applications Features}

The most important feature of Basirah include:

- Runs on iOS devices.

- Both requesters and volunteers maintain accounts and profiles in the application.

- Categories are shown to help choosing from a list of requests.

- Requesters can request any type of readable documents.

- Requesters can request unlimited number of audio records to favorite documents. 
- Both requesters and volunteers can organize and save their library of requests.

The application is intended to be used by users who are visually impaired requesters and nonvisually impaired volunteers and is not limited to certain age or gender. Nevertheless, since it is a mobile application, it does require a certain knowledge of using smart phones. Visually impaired interactions are supported in iOS with the use of VoiceOver [4].

\section{System Design}

The system consists of the mobile application with embedded database and the execution environment. The software system is comprised of three main components (see Fig. 4):

- The issuing request components contains functions related to imitating the requests, receiving audio links that respond to the request, and deleting requests and responses.

- The request handling component contains functions related to filtering requests by categories based on the users' area of interest and language.

- The last component is the users' services that handles functions related to users such as displaying and editing their profiles, sign up, $\log$ in, and $\log$ out.

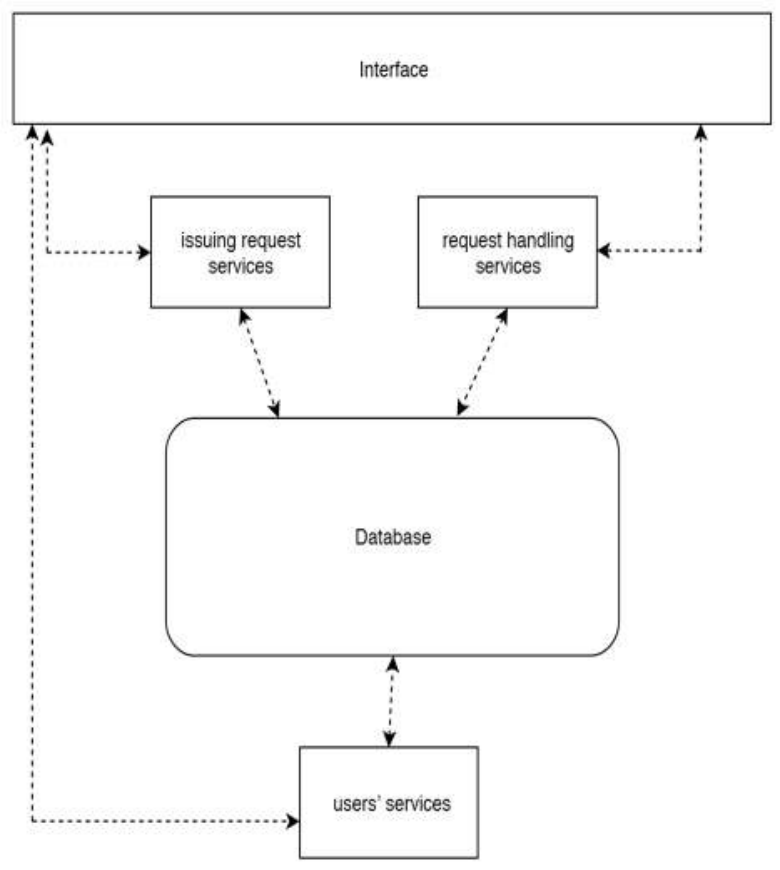

Figure 4. Basirah system architecture. 


\section{System Implementation}

The application was developed using Xcode for iOS devices [5]. A local database was used to store the application's required information. For that purpose, Realm database was utilised as an alternative to SQLite that is relatively faster to setup and use [6][7]. The initial version of the application is coded in English, with future plans to expand to Arabic and other language.

The user interface was designed to be user friendly and accessible to users who are visually impaired and sighted volunteers. The first screen in Basirah is the login screen (see Fig. 5), where both volunteers and visually impaired users can login to their personal accounts. If the application is new to the account, it is possible to register via the sign-up screen shown in Fig. 6. The login page has two fields: username and password. Alternatively, the sign-up screen requests the new user's name, unique username, email, password, and role as volunteer or audio requester.

Volunteers and requesters do not share the same home screen. If the user is a volunteer, the home screen displays the list of requests available that can be filtering. Filtering will allow the volunteer to display requests that are of their direct interest. Requests are filtered by the request category and language as shown in Fig. 7. After selecting the desired parameters, the list of matched request are displayed with an 'accept' button next to each request. This button is hidden and only appears when dragging to the left as shown in Fig. 8. Volunteers are also able to view their profiles as shown in Fig. 9. From that page, the volunteer can choose to log out and sign out of their profiles. Another option provided to volunteers allows them to edit their profile as displayed in Fig. 10. Furthermore, volunteers can view the requests they have accepted, past and present, which is displayed in a new window (see Fig. 11). To submit an audio recording link for a request, the volunteer navigates the list of the request and clicks on the desired request. This action triggers the system to display a second page of a text field for the volunteer to share the link to the audio recording as seen in Fig. 12.

The second type of user to the system with a dedicated home screen is the user who is visually impaired. This home screen displays the user's profile and four buttons linked to four different actions: log out, edit profile, new request, and my requests (see Fig. 13). The log out and edit profile options offers the same functionality is seen before with the volunteer user. To enter a new request, the visually impaired user will click on 'new request' that opens to a new interface that prompts the user for the title of the request, its category, language, and deadline. This window is illustrated in Fig. 14. After submitting the request, the user is then taken back to the home screen. The visually impaired user is able to view all of their requests by clicking on 'my requests'. In this window, a list of all requests is shown and each request is appended with a status. As seen in Fig. 15 and 16, clicking on any requests opens a new window with the link the audio recording provided by one of the application's volunteers.

\section{System Testing}

Several testing approaches were undertaken to ensure the integrity and validity of the application. Each unit was first testing separately, then integration testing was carried out, and finally the functionality of the system was tested as a whole. 
By testing the unit separately, we are able to pinpoint the problem early as they occur. In integration testing, events that required multiple components (i.e. units) were tested. During this test, linked windows were tested to ensure that each button links to the correct page and the functionalities is working as expected. Minor issues relating to request were identified in this phase, but has since been solved. The system was then evaluated as a whole by examining the overall functionalities and ensuring that the requirements were implemented.

\section{Conclusions}

Reading is an important literacy skill that is often obtained from childhood. People who are visually impaired are disadvantaged due to their loss of sight and must rely on other senses to compensate. Several application and products aims to facilitate the reading process for users who are visually impaired as was reported in Section 2. Nevertheless, more support is still need as improvements are required to satisfy their needs and ease accessibility to this part of the community. This paper addressed this problem by developing a mobile application that brings the community together.

The developed application, Basirah, gives sighted community members the opportunity to volunteer and help visually impaired members of their community. Users who are visually impaired submit audio requests that are accepted and provided by volunteers. For future work, we intend to allow people who are visually impaired to rate the provided audio recording. We also plan to allow the requester to specify unique properties they expect in the audio recording.

\section{References}

[1] Mahmoud El-Gayyar, Amira Ibrahim, Ahmed Sallam, "The ArSL keyboard for android", Intelligent Computing and Information Systems (ICICS) 2015 IEEE Seventh International Conference on, pp. 481-486, 2015.

[2] "Read2Go | Bookshare", Bookshare.org, 2018. [Online]. Available: https://www.bookshare.org/cms/help-center/reading-tools/read2go. [Accessed: 02- May2018].

[3] " [كتبة المنارة", International Manarah Library, 2018. [Online]. Available: https://arabcast.org/?mod=page\&ID=3. [Accessed: 17- Apr- 2018].

[4] "Vision accessibility", Apple, 2018. [Online]. Available: https://www.apple.com/sa/accessibility/iphone/vision/ [Accessed: 26- Aug- 2018].

[5] "Xcode", Apple Developer, 2018. [Online]. Available: https://developer.apple.com/xcode/. [Accessed: 26- Aug- 2018].

[6] "Realm Studio: open, edit, and manage your Realm data", Realm.io, 2018. [Online]. Available: https://realm.io/products/realm-studio/. [Accessed: 06- Apr- 2018].

[7] "Edge Sync Mobile Platform \& Database | Realm", Realm.io, 2018. [Online]. Available: https://realm.io/docs/swift/latest/. [Accessed: 13- Feb- 2018]. 


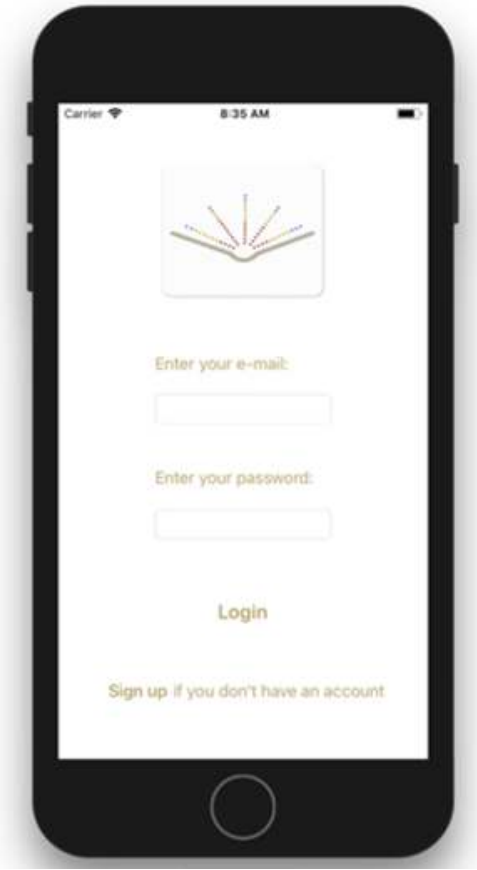

Figure 5. Login window.

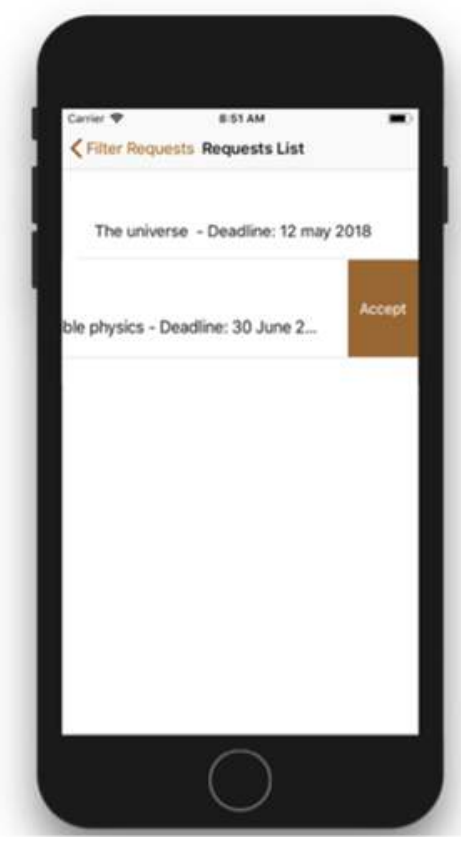

Figure 8. Accept requests window.

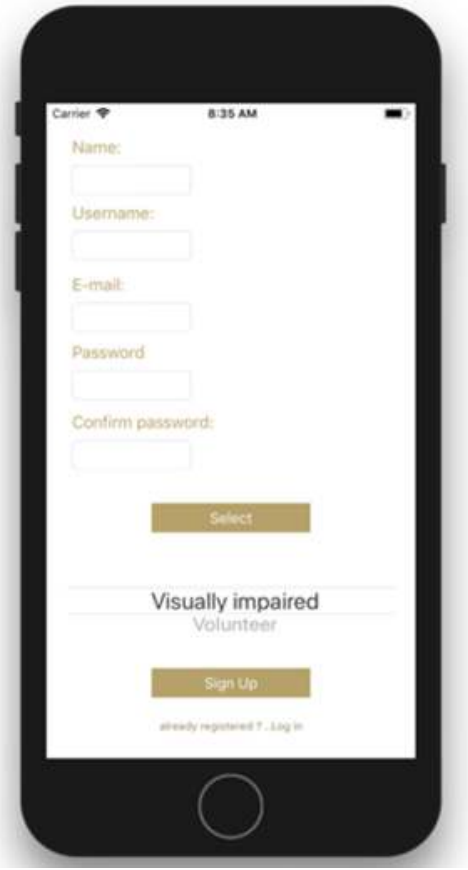

Figure 6. Sign up window.

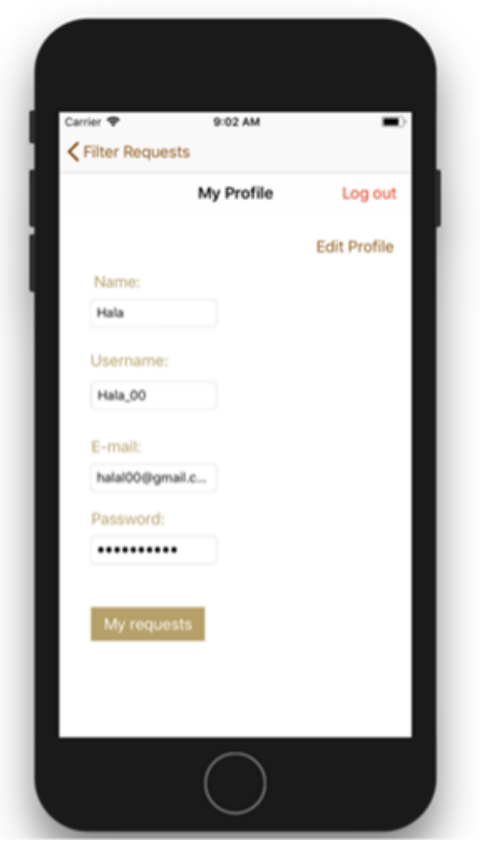

Figure 9. Volunteer profile window.

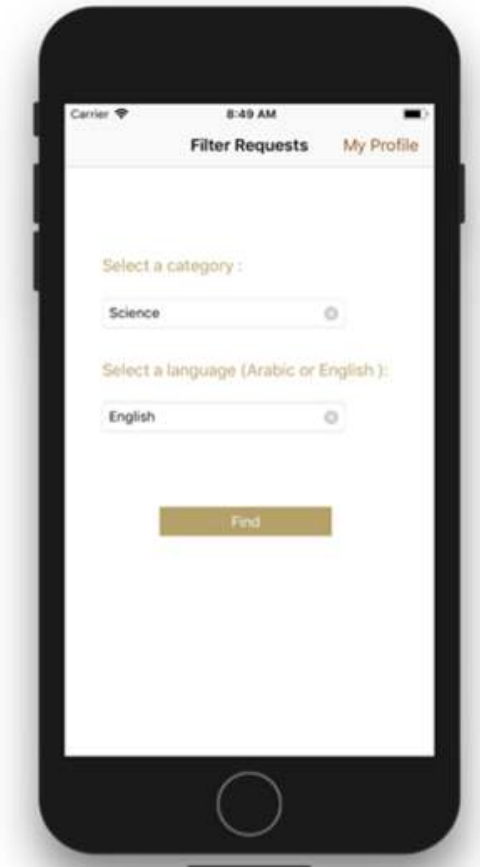

Figure 7. Filtering requests window.

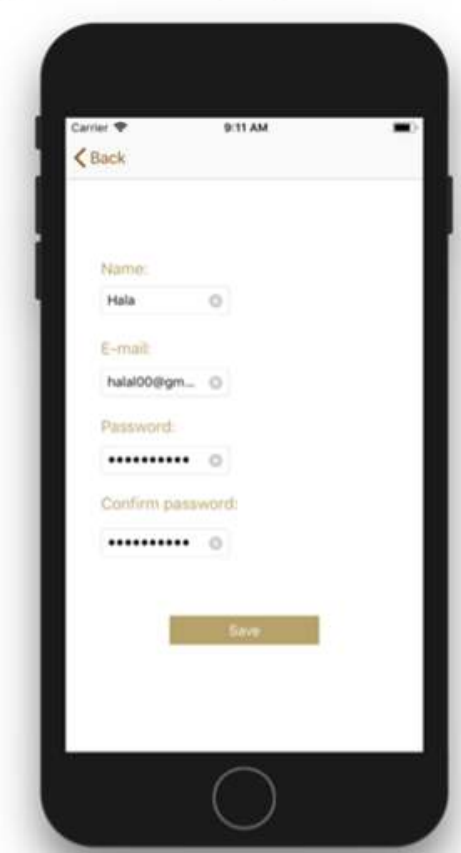

Figure 10. Edit profile window 


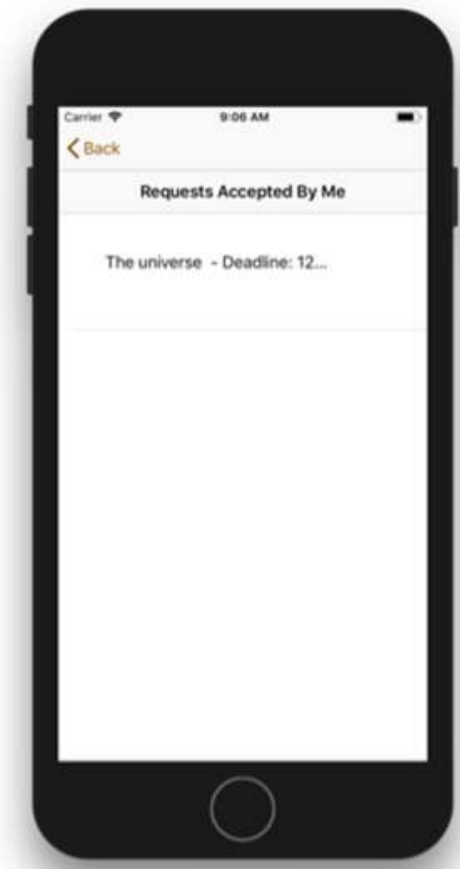

Figure 11. Volunteer's list of accepted requests.

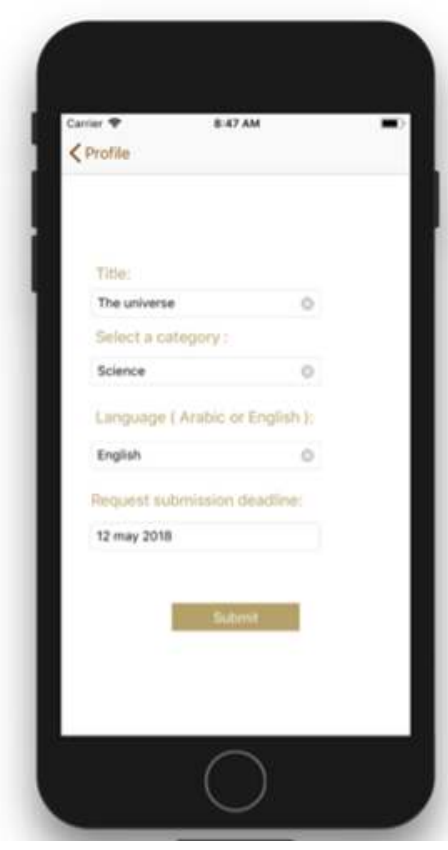

Figure 14. New request window.

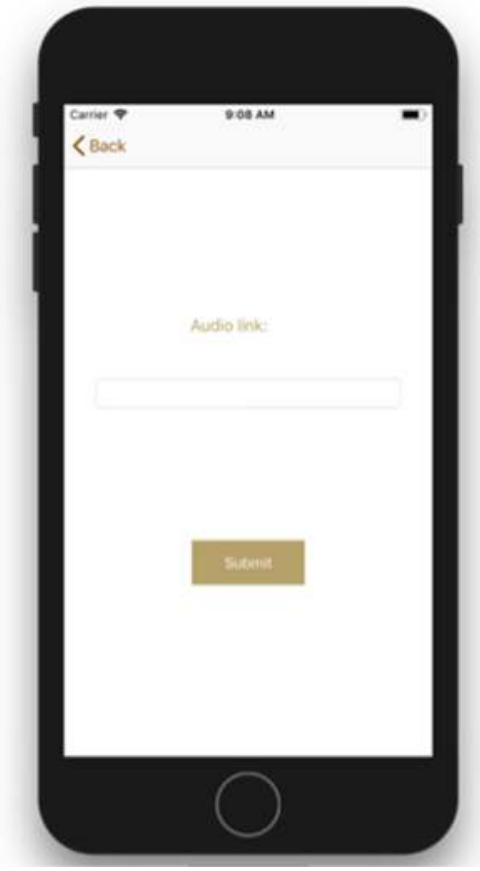

Figure 12. Submit audio link window.

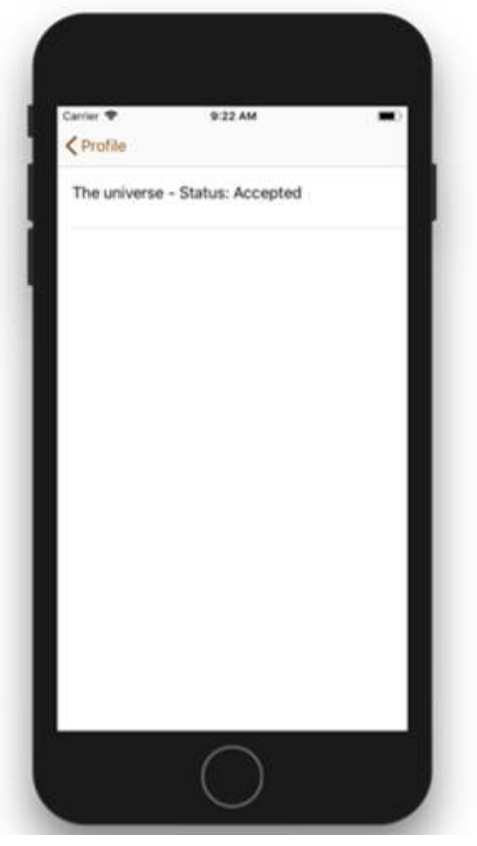

Figure 15. Visually impaired user list of requests window.

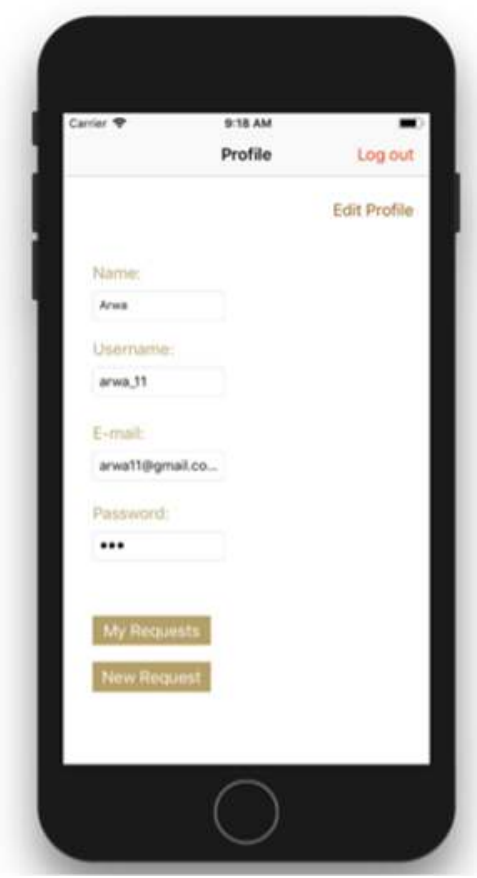

Figure 13. Visually blind user's profile page.

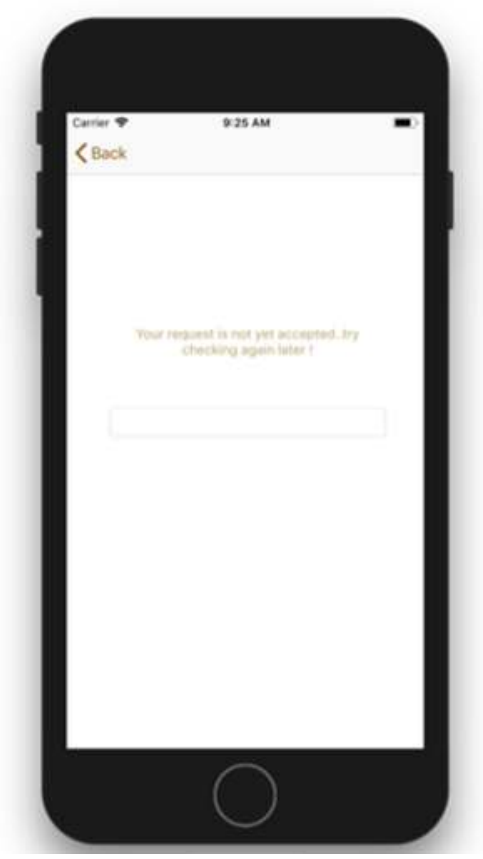

Figure 16. Request link status window. 